Tecnología y

Ciencias $\stackrel{\Xi}{\triangleleft}$ gua
2020, Instituto Mexicano de Tecnología del Agua

Open Access bajo la licencia CC BY-NC-SA 4.0

(https://creativecommons.org/licenses/by-nc-sa/4.0/)

DOI: $10.24850 / \mathrm{j}$-tyca-2020-04-10

Notas

\title{
EI empleo de cimacios tipo WES en vertedores tipo \\ laberinto. Parte II
}

\section{The employment of ogees type WES in spillways type labyrinth. Part II}

Rafael Pardo-Gómez ${ }^{1}$

${ }^{1}$ Centro de Investigaciones Hidráulicas, Universidad Tecnológica de La Habana, La Habana, Cuba, rpardo@cih.cujae.edu.cu

Autor para correspondencia: Rafael Pardo-Gómez, rpardo@cih.cujae.edu.cu

\section{Resumen}

Los vertedores tipo laberinto son relativamente novedosos. Su empleo comenzó a mediados del pasado siglo, siendo la década de 1990 y comienzos de 2000 cuando se desarrolló la mayor cantidad de investigaciones a escala de laboratorio sobre tal tipo de vertedores. En el presente trabajo se muestran los resultados de investigaciones experimentales dirigidas a definir y obtener las herramientas para el empleo de cimacios tipo WES en ese tipo de vertedores, dadas sus 
ventajas en cuanto al coeficiente de gasto, en comparación con otros tipos de estructuras. Se presentan los resultados de estudios a escala de laboratorio en modelo tridimensional relativos al empleo del concepto de longitudes características de estas estructuras para su empleo en los vertedores de laberinto, así como de la longitud mínima de los ápices de entrada, con lo cual se eliminan la interferencia de láminas y sumergencia en dichas estructuras.

Palabras clave: vertedores de laberinto, tipos de cresta del vertedor, cimacios WES truncados, coeficientes de contracción de la red de flujo, longitud mínima del ápice de entrada.

\section{Abstract}

Labyrinth type weirs are relatively new as their employment started by the middle of the past century. The nineties decade and beginning of the present century have witnessed a great deal of laboratory scale research about this type of weirs. In the present work experimental research results are shown to evaluate the possibilities of employing type WES ogees within the labyrinth type weirs given the advantage of a better discharge coefficient as related to other ogee types. Results from tridimensional laboratory scale models are presented relative to the use of characteristics length in order to allow their use in labyrinth spillways.

Keywords: Labyrinth weirs, weir crest types, WES ogees, truncated ogees, weir with ventilated sheet, weir with non-ventilated sheet.

Recibido: 28/03/2029 
Tecnología y

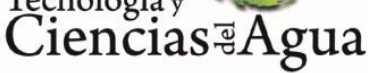

2020, Instituto Mexicano de Tecnología del Agua

Open Access bajo la licencia CC BY-NC-SA 4.0

(https://creativecommons.org/licenses/by-nc-sa/4.0/)

Aceptado: $28 / 10 / 2019$

\section{Introducción}

En la Figura 1 se presenta un esquema en el que se introducen las variables hidráulicas y geométricas de los aliviaderos con configuración en laberinto (Crookston, 2010).

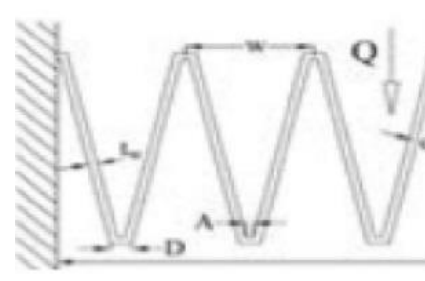

PLANTA
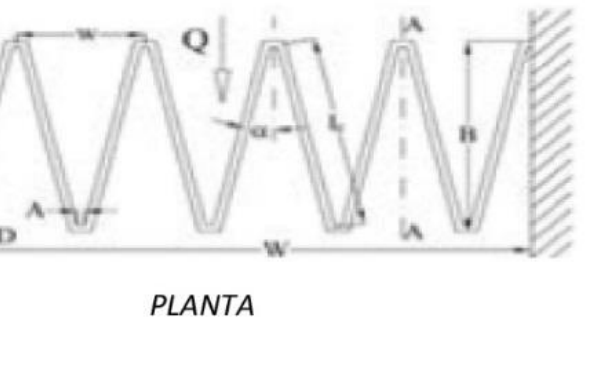

\begin{abstract}
A
\end{abstract}

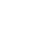

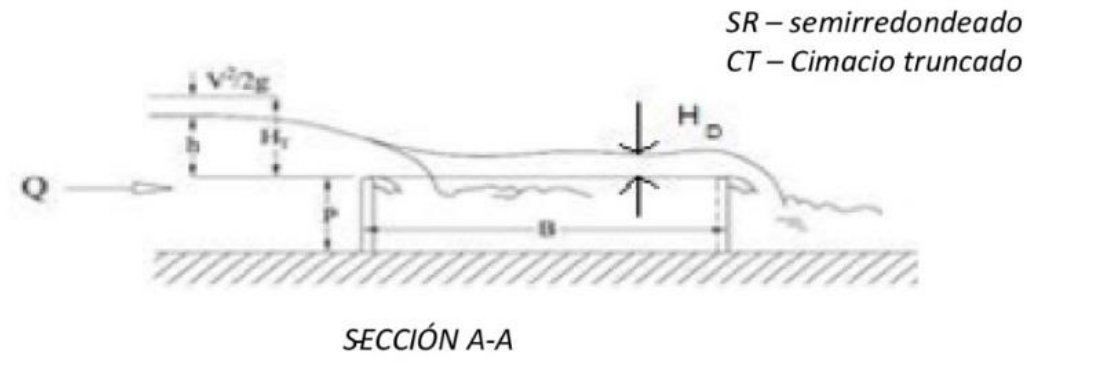

Figura 1. Esquema de un vertedor tipo laberinto. Fuente: Crookston (2010). 
Como se demostró en el artículo anterior (parte I) (Pardo, 2019), el empleo de cimacios WES truncados en vertedores tipo laberinto resulta atractivo por presentar coeficientes de gasto con valores superiores a los de otros tipo de vertedores, que es totalmente válido, pues no se afecta la descarga (entiéndase el coeficiente de gasto), lo que permitiría diseñar estos cimacios, empleando para ello las mismas herramientas que para el caso de los frontales (Pardo, 2019).

A partir de las consideraciones expresadas en el párrafo anterior, en el laboratorio de modelos físicos del Centro de Investigaciones Hidráulicas ( $\mathrm{CIH})$, de la Universidad Tecnológica de La Habana "José Antonio Echeverría" (CUJAE), se ha continuado con la serie de experimentos para facilitar el empleo de los referidos cimacios en los vertedores tipo laberinto.

El primer estudio experimental consistió en identificar la influencia del truncado en esos tipos de cimacios en el valor de sus respectivos coeficientes de gasto; los resultados se presentaron en una publicación anterior (Pardo, 2019). La segunda etapa de investigaciones se enfocó en el empleo del concepto de longitudes características de estos vertedores para su empleo en aliviaderos con configuración en laberinto y a este aspecto se dedica el presente trabajo.

Se definen como objetivos principales de la investigación:

1. Estudiar el comportamiento del coeficiente de gasto de un vertedor tipo WES frontal cuando se emplea en un vertedor con configuración en laberinto. 
2. Determinar las variables a considerar para emplear los conceptos de longitudes características de un vertedor WES, cuando se emplea en un vertedor con configuración en laberinto.

\section{Detalles de la instalación del laboratorio, objetivos de la investigación y diseño del modelo}

Para la puesta en funcionamiento del modelo, el Centro de Investigaciones Hidráulicas $(\mathrm{CIH})$ cuenta con un laboratorio especializado, en el cual se encuentran seis plazas, una de ellas, en la que se va a desarrollar la investigación; es rectangular, con una longitud de $15 \mathrm{~m}$, ancho de $2.85 \mathrm{~m}$ y altura de $2 \mathrm{~m}$; además, tiene un canal de salida aguas abajo de dicha plaza para guiar el agua hacia un vertedor de aforo que se encuentra al final con vertimiento libre. Esta plaza posee una entrada de agua proveniente de un tanque de carga constante y con caudal de hasta $1 \mathrm{~m}^{3} / \mathrm{s}$, en la que se llena un estanque de $2.85 \mathrm{~m}$ de longitud por $1 \mathrm{~m}$ de ancho y $1.5 \mathrm{~m}$ de altura (Figura 2). 


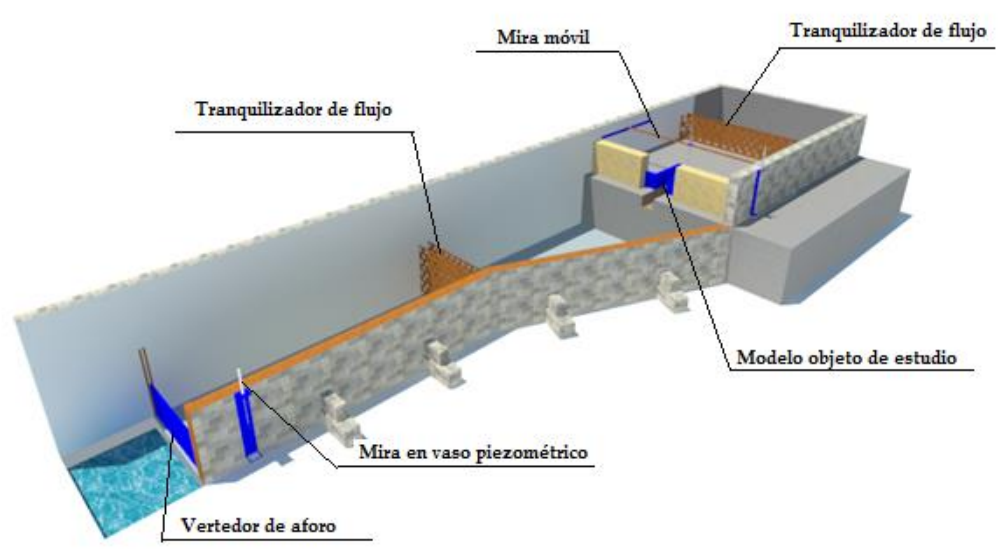

Figura 2. Vista general de la plaza de trabajo. Fuente: Pagés-Lamas (2017).

\section{Equipos utilizados en la investigación}

- Bomba de agua.

- Válvula de mando eléctrico para garantizar el rango de gastos.

- Dos tranquilizadores de flujo con agujeros de $3 \mathrm{~cm}$ de diámetro y espaciados a $5 \mathrm{~cm}$.

- Rejilla de madera a la salida del disipador para disminuir la turbulencia en la superficie.

- Dos miras de punta graduadas hasta la décima de milímetro; una de ellas es móvil. 
- Nivel de topógrafo para nivelar el modelo en forma general y tomar los ceros tanto al vertedor de laberinto como al vertedor de aforo.

\section{Ubicación del modelo}

Al inicio del área de trabajo se colocó un disipador de energía para tranquilizar el flujo que viene de la válvula hacia la plaza de ensayo. El vertedor de laberinto se ubicó a $2.5 \mathrm{~m}$ aguas abajo de dicho disipador de energía; $0.5 \mathrm{~m}$ aguas abajo tiene una caída libre, vertiendo en un canal, donde se colocó otro disipador de energía similar al anterior y se le adicionó una rejilla flotante de madera para tranquilizar el flujo en el canal de salida de $1.32 \mathrm{~m}$ de ancho, donde se ubicó un vertedor de aforo rectangular al final con caída libre para medir los gastos. También se colocaron dos miras de punta, una de ellas en el vaso piezométrico ubicado en el vertedor de aforo, para medir las cargas sobre dicho vertedor; la otra sobre un raíl móvil para medir la carga estática sobre el vertedor de laberinto (Figura 2).

\section{Etapas del procedimiento experimental}


Se establecen las etapas siguientes:

1. Diseño del vertedor de aforo rectangular.

2. Diseño y construcción del modelo a estudiar.

3. Mediciones y resultados experimentales.

\section{Etapa 1. Diseño del vertedor de aforo rectangular}

El vertedor de aforo presente en la instalación es el mismo construido por Pagés (Pagés-Lamas, 2017), al cual se disminuyó su longitud, ya que resultaba excesiva para la presente investigación y, sobre todo, porque ello permite dimensionar un vertedor sin contracciones laterales (Figura 3).

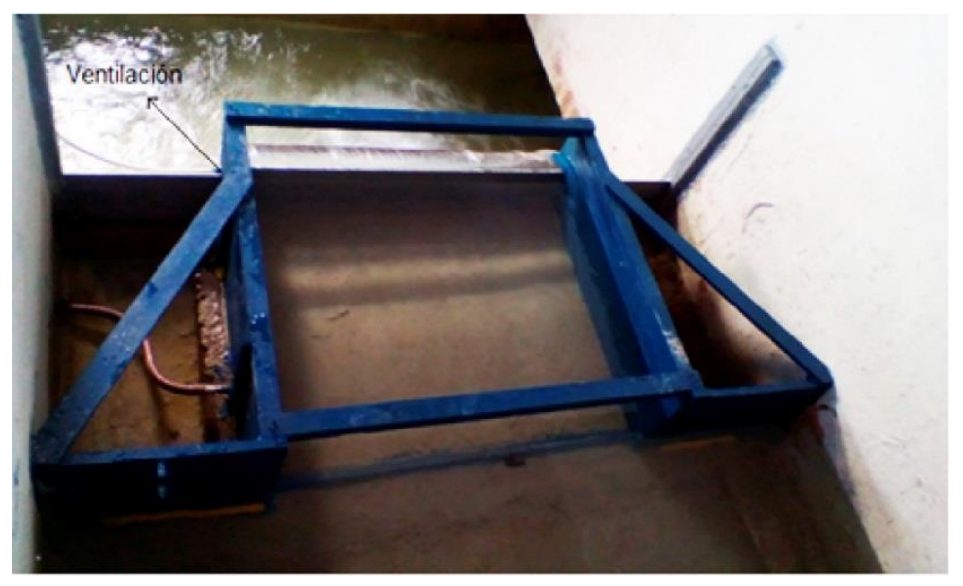


Tecnología y

Ciencias $\stackrel{\Im}{\Im}$ Agua
2020, Instituto Mexicano de Tecnología del Agua

Open Access bajo la licencia CC BY-NC-SA 4.0

(https://creativecommons.org/licenses/by-nc-sa/4.0/)

Figura 3. Vertedor rectangular de caída libre y sin contracciones laterales. Fuente: Borroto-Roche (2018).

\section{Etapa 2. Diseño y construcción del modelo a estudiar}

A partir de los objetivos de la investigación, se diseñó el modelo a emplear, siguiendo los siguientes pasos:

Definición del cimacio a emplear. Se seleccionó un cimacio tipo WES truncado, que se diseñó para una carga de $7.2 \mathrm{~cm}$ y una altura de $20 \mathrm{~cm}$, las mismas medidas empleadas en la investigación realizada por Carralero (Carralero, 2016) y Pagés (Pagés-Lamas, 2017), para los cimacios frontales y en laberinto, respectivamente. Además, se realizó el truncado para una relación de $X / X_{t}=0.2$, ya que es el mínimo valor que se le puede dar a los cimacios WES para que no se afecte su curva de capacidad de servicio (Carralero, 2016) (Figura 4). 
Tecnología y

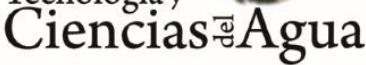

2020, Instituto Mexicano de Tecnología del Agua

Open Access bajo la licencia CC BY-NC-SA 4.0

(https://creativecommons.org/licenses/by-nc-sa/4.0/)

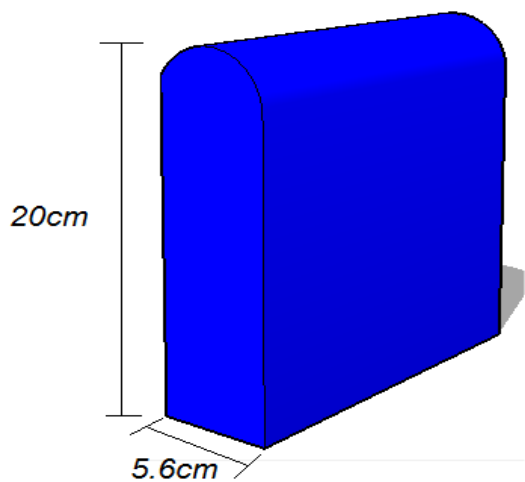

Figura 4. Diseño del cimacio WES truncado $X / X_{t}=0.2$. Fuente: PagésLamas (2017).

- Definición de la configuración en planta del modelo para cumplir el primer objetivo arriba expuesto, en particular en lo relativo a eliminar la interferencia de láminas. El diseño del aliviadero de laberinto se realizó de forma rectangular y consta de un solo ciclo para llevar a cabo toda la investigación (Figura 5).

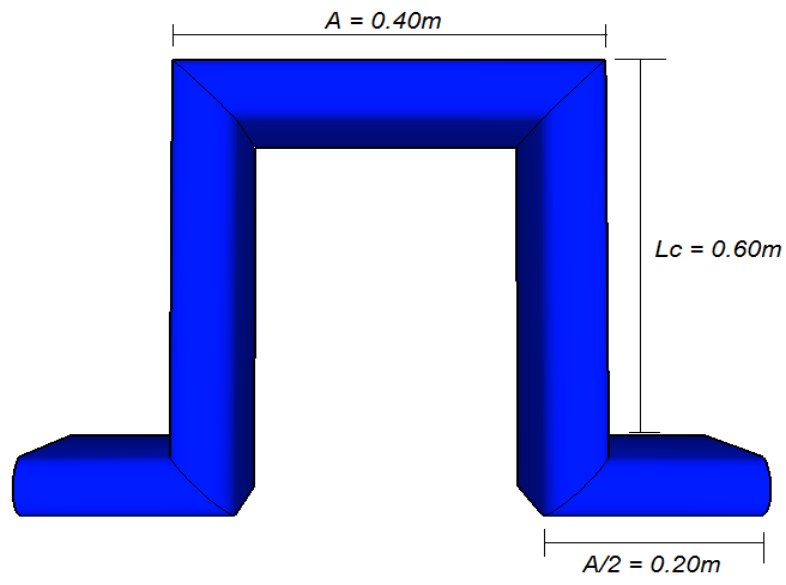

Figura 5. Vista en planta del modelo de vertedor de laberinto. Fuente: Pagés-Lamas (2017). 
En el modelo físico seleccionado, la red de flujo hacia el mismo se puede representar en forma simplificada, según se muestra en la Figura 6, la cual tiene dos situaciones definitivamente tridimensionales diferentes entre sí: una es en los extremos del ápice de entrada y la otra en los extremos del ápice de salida; a continuación se reflexiona acerca de ambas por separado.

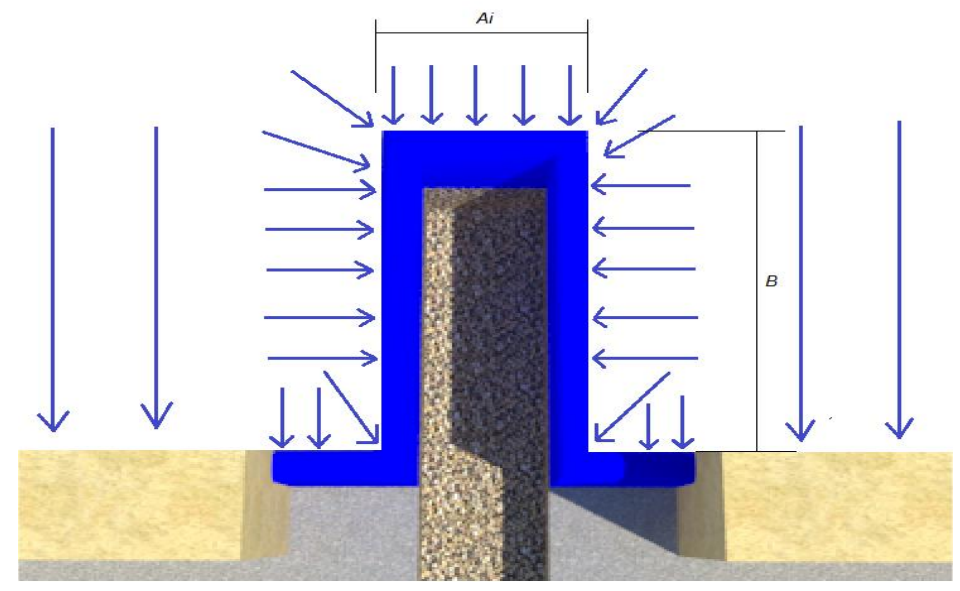

Figura 6. Red de flujo de llegada al laberinto. Fuente: Pagés-Lamas (2017).

- Respecto al ápice de entrada. La descarga del flujo sobre los extremos del ápice de entrada es convergente y tiene lugar total interferencia entre la lámina que vierte sobre el ápice $A_{i}$ y la que descarga sobre el lado lateral del ciclo $B$ en las proximidades de cada extremo y que resulta inevitable, es decir, es consustancial a esta configuración. Dichas zonas se destacan en color rojo en la 
Figura 7; mientras que en la porción central del ápice superior que se conserva en color negro en esta figura no hay interferencia de láminas siempre que la magnitud $A_{i}$ sea lo suficientemente alta como para mantener alejadas las descargas por los lados B entre sí.

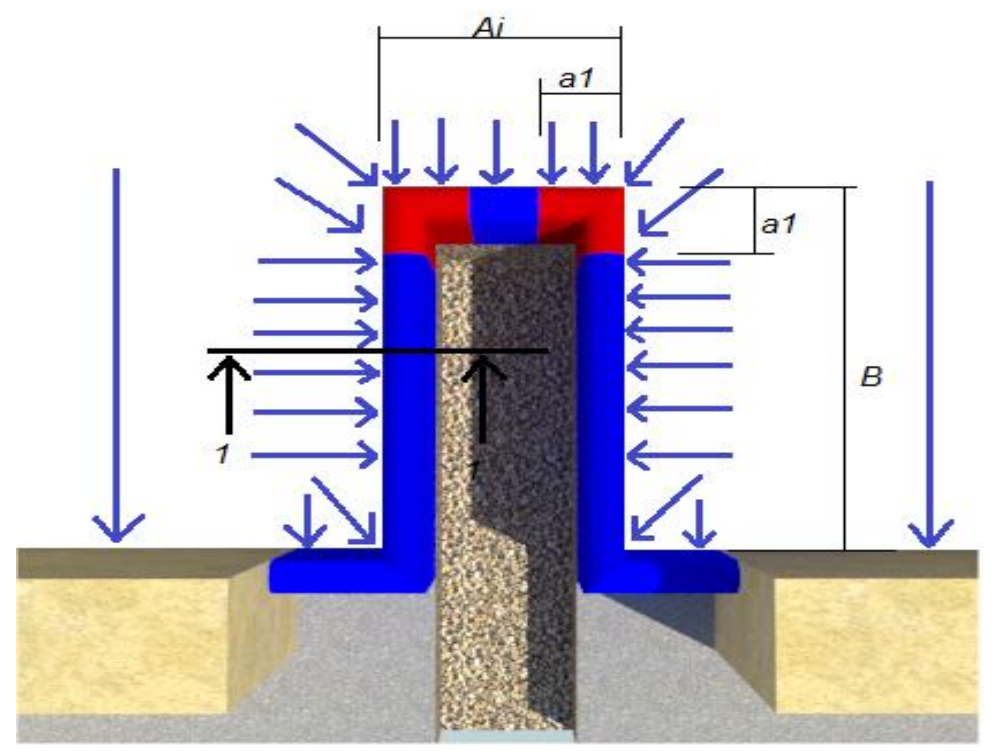

Figura 7. Zonas de ocurrencia de interferencias de láminas inevitables (destacadas en color rojo). Fuente: Pagés-Lamas (2017).

La magnitud $a_{1}$-que identifica la longitud de interferencia de lámina inevitable asociada con cada extremo del ápice de entrada- es posible definirla como aquella franja del cimacio que es igual a la longitud externa del chorro que descarga sobre el cimacio, según se indica en la Figura 8. 
Tecnología y

Ciencias $₫$ Agua
2020, Instituto Mexicano de Tecnología del Agua

Open Access bajo la licencia CC BY-NC-SA 4.0

(https://creativecommons.org/licenses/by-nc-sa/4.0/)

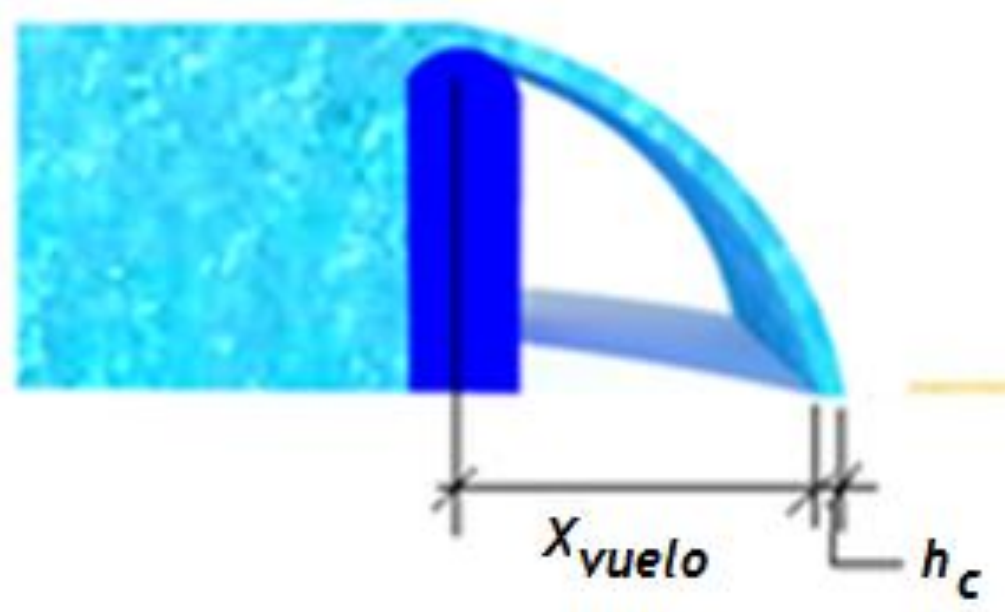

Figura 8. Longitud de vuelo externa del chorro desde el extremo de aguas arriba del cimacio hasta el nivel de la solera en el ciclo de salida, Xvuelo. Sección transversal 1 -1 indicada en la Figura 7. Fuente: PagésLamas (2017).

\section{Esto es:}

$$
a_{1}=X_{\text {VUELO }}
$$

A partir de lo expresado, se puede inferir que el valor mínimo de la longitud del ápice de entrada se puede definir como:

$$
A_{i-\min }=2 a_{1}
$$

Por lo tanto, un valor inferior al expresado en la Ecuación (2) implicaría que los chorros de la descarga desde los laterales del ciclo 
chocarían entre sí, esto es, producirían interferencia de láminas adicional a la ya llamada inevitable, como se comentó.

- Con respecto al ápice de salida. En este caso no se produce interferencia de láminas en la descarga sobre el cimacio, pues la salida sobre el lateral es divergente con relación a la descarga sobre el ápice. Aquí, el fenómeno que ocurre tiene lugar antes de iniciar el flujo sobre el cimacio, es decir, en el flujo de aproximación al cimacio. Una explicación al fenómeno físico es la siguiente: una gota de agua ubicada en el punto $O$ indicado en la Figura 9, que se describe como un punto equidistante al cimacio lateral y al ápice de salida, y sobre la diagonal que marca la unión entre dichos cimacio lateral y el referido ápice, puede seguir su trayectoria siguiendo una de las opciones que se indican a continuación:

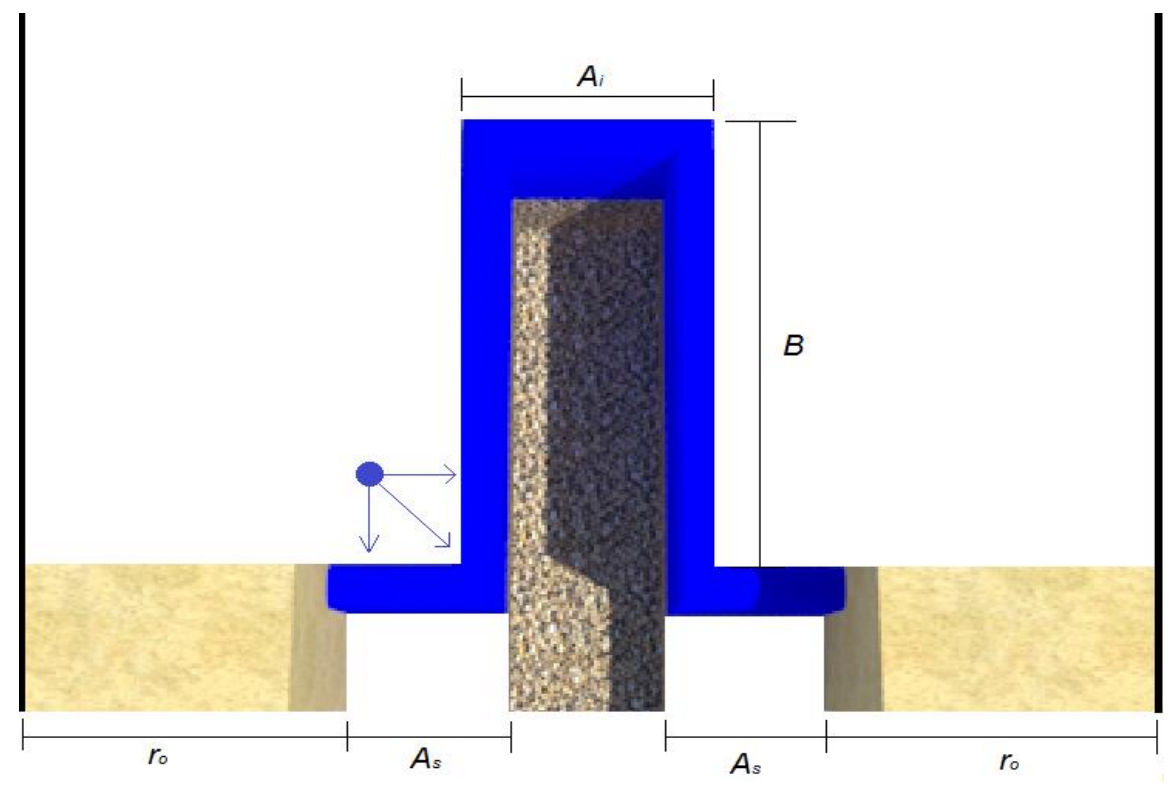

Figura 9. Acerca del ápice de salida. Fuente: Pagés-Lamas (2017). 
1. Perpendicular al cimacio lateral, en caso de que la componente de la velocidad de aproximación en ese sentido sea la predominante.

2. Perpendicular al cimacio del ápice, en caso de que la componente de la velocidad en ese sentido sea la predominante.

3. Por una línea intermedia entre la dos anteriores, de preferencia sobre la mencionada unión cuando la velocidad del flujo en la aproximación sea baja y sin predominio de una componente sobre la otra.

La ocurrencia de los casos 1 y 2 representan una reducción de la capacidad de descarga de uno de los lados; esto es, si es el caso 1, se afectará la capacidad de descarga sobre el ápice; mientras que si es el caso 2 , entonces la reducción de la capacidad de descarga será la del cimacio lateral.

El caso 3 representa la no afectación de la descarga, al lograrse buena aproximación a la descarga en todos los sentidos; ello se logra garantizando suficiente separación entre dos ciclos consecutivos del laberinto, o entre los ciclos de los extremos y la pared lateral del canal de aproximación.

Dado que lo antes expuesto indica la presencia de interferencia de láminas sólo debida a los extremos del ápice de entrada para la

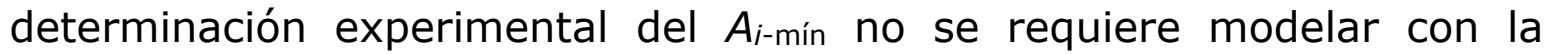
presencia de ápices de salida, por lo que se continuarán los ensayos, según el esquema que se muestra en la Figura 10. 


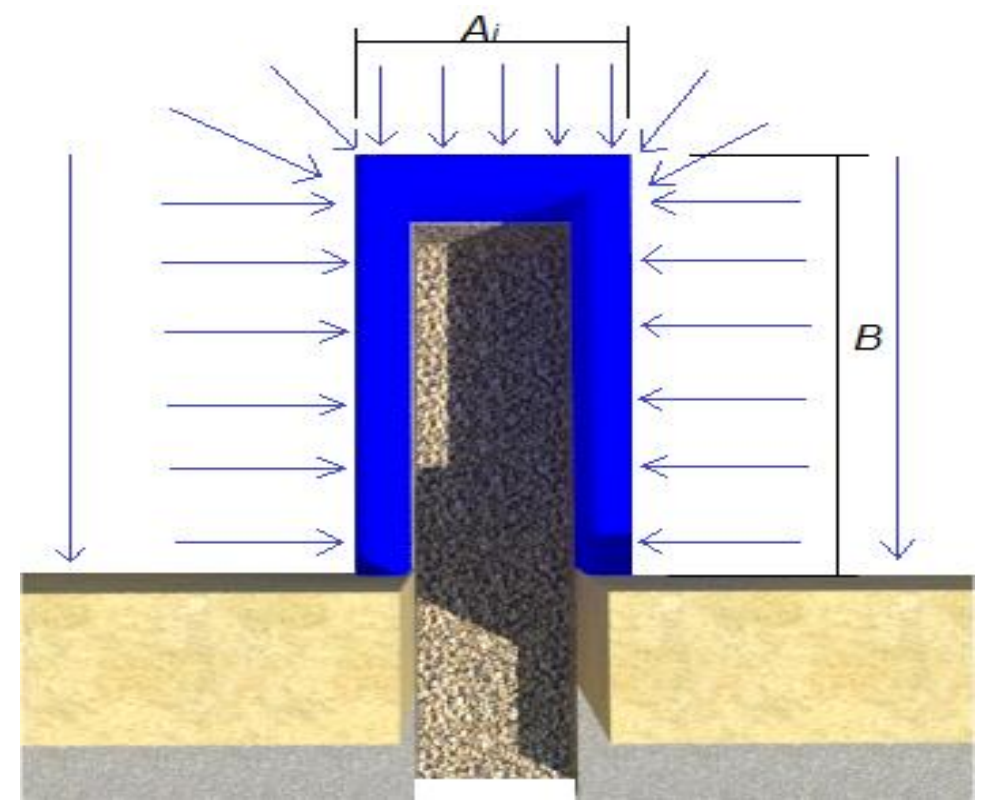

Figura 10. Esquema de la instalación para determinar el $A_{\text {mín }}$ (ancho mínimo del ápice de entrada). Fuente: Pagés-Lamas (2017).

Una hipótesis a partir de lo aquí expuesto y que posteriormente se valida mediante los estudios experimentales que se desarrollan, es que la construcción de un vertedor de configuración equipado con vertedor tipo WES truncado tendrá el mismo coeficiente de gasto que un vertedor frontal, al actuar con las mismas condiciones hidráulicas (gasto y carga hidráulica), siempre que los ápices de entrada sean de longitud igual o mayor que la longitud mínima que se determine en los estudios experimentales.

- Definición de la configuración en planta del modelo para cumplir el segundo objetivo arriba expuesto. Hasta el presente los vertedores empleados con frecuencia con configuración en laberinto han sido los de cresta un cuarto 
Tecnología y

Ciencias $\stackrel{\Im}{\Im}$ Agua
2020, Instituto Mexicano de Tecnología del Agua

Open Access bajo la licencia CC BY-NC-SA 4.0

(https://creativecommons.org/licenses/by-nc-sa/4.0/)

redondeada y los semirredondeados; en ellos, la longitud vertedora medida a lo largo de la cresta $\left(L_{c}\right)$ es igual a la longitud medida a lo largo del paramento superior $\left(L_{p}\right)$ debido al espesor pequeño de estos vertedores. Como se ha apreciado en diversas publicaciones sobre el tema (Tullis, Asce, Young, \& Chandler, 2007; Crookston, 2010), mediante estudios experimentales se ha obtenido el coeficiente de gasto de ellos $\left(C_{d}\right)$, que satisface la Ecuación (3).

$Q=\frac{2}{3} C_{d} L_{c} \sqrt{2 g} H_{T}^{3 / 2}$

En la que $H_{T}$ es la carga hidráulica total actuante sobre la cresta del vertedor.

Es oportuno destacar que el mencionado coeficiente $C_{d}$ es afectado por la modificación de la red de flujo que implica la configuración en laberinto (Figura 6), y que la longitud $L_{c}$ es la longitud total y no la longitud efectiva $(L)$, que es la más comúnmente utilizada en aliviaderos (Pardo \& Alegret, 2012).

Al introducir el empleo de los cimacios WES truncados en los vertedores de configuración en laberinto, Pardo (2019) considera oportuno sustituir la Ecuación (3) por la Ecuación (4) que se presenta a continuación, por ser de mayor empleo en aliviaderos:

$Q=m L \sqrt{2 g} H_{o}^{3 / 2}$ 
Tecnología y

Ciencias $₫$ Agua
2020, Instituto Mexicano de Tecnología del Agua

Open Access bajo la licencia CC BY-NC-SA 4.0

(https://creativecommons.org/licenses/by-nc-sa/4.0/)

Donde $H_{0}$ es la carga hidráulica total sobre la cresta del vertedor y $m$ es el coeficiente de gasto, sin considerar la presencia de la modificación a la red de flujo antes mencionada. El empleo de esta longitud efectiva impone establecer la relación entre ella y la longitud $L_{c}$, para ello se hace uso de la Figura 11.

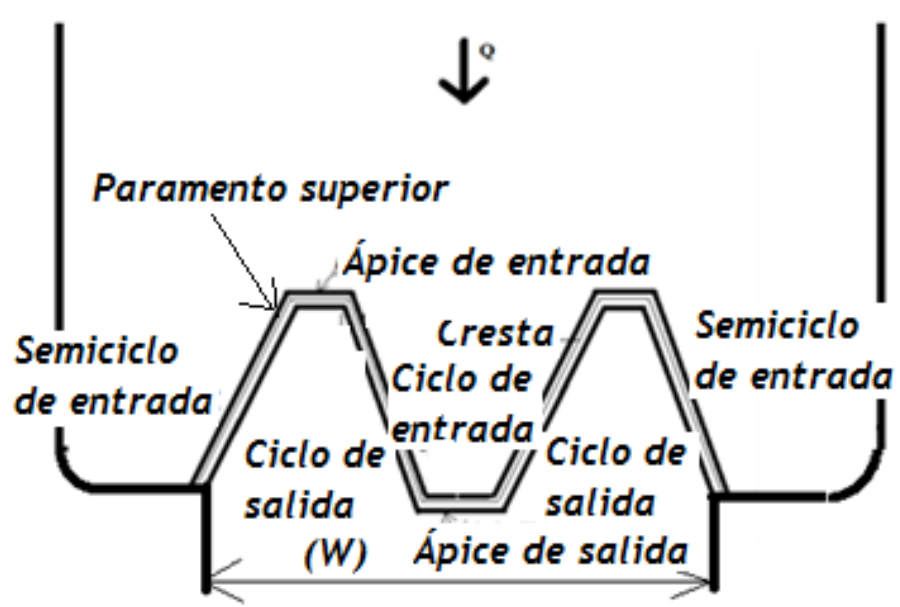

(a)

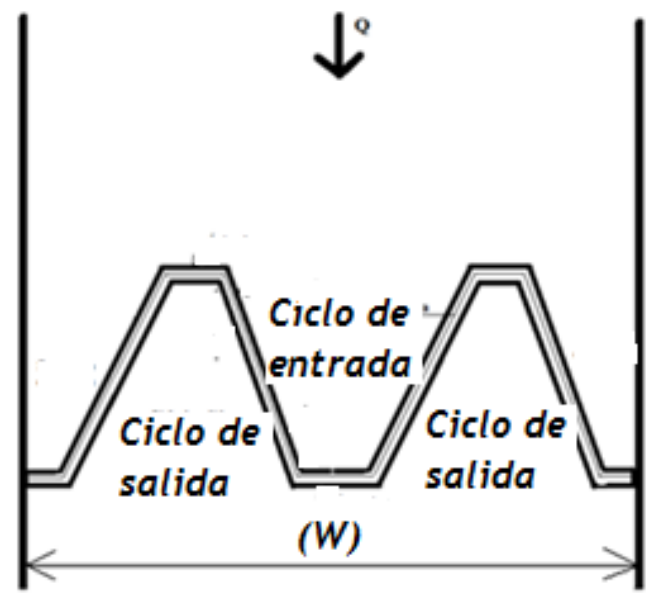

(b)

Figura 11. Esquema de un vertedor en laberinto de dos ciclos y ángulo central $a$; a) sin semiápices de salida; b) con semiápices de salida.

Estas longitudes se relacionan entre sí de la manera que se presenta a continuación.

- Las longitudes $L_{P}$ y $L_{c}$ según relaciones geométricas:

$L_{c}=L_{P}-(j-k)\left[2 X_{c}\left(\frac{1}{\cos \alpha}-\operatorname{tg} \propto\right)\right]$ 
Tecnología y

Ciencias $\stackrel{\Im}{\Im}$ Agua
2020, Instituto Mexicano de Tecnología del Agua

Open Access bajo la licencia CC BY-NC-SA 4.0

(https://creativecommons.org/licenses/by-nc-sa/4.0/)

Siendo:

$j:$ total de inflexiones de entrada.

$k$ : total de inflexiones de salida.

$a$ : ángulo que forma el cimacio vertedor lateral del ciclo con el eje longitudinal según se indica en la Figura 11.

$X_{c}$ : distancia desde el paramento superior a la cresta del cimacio (ver la figura 12).

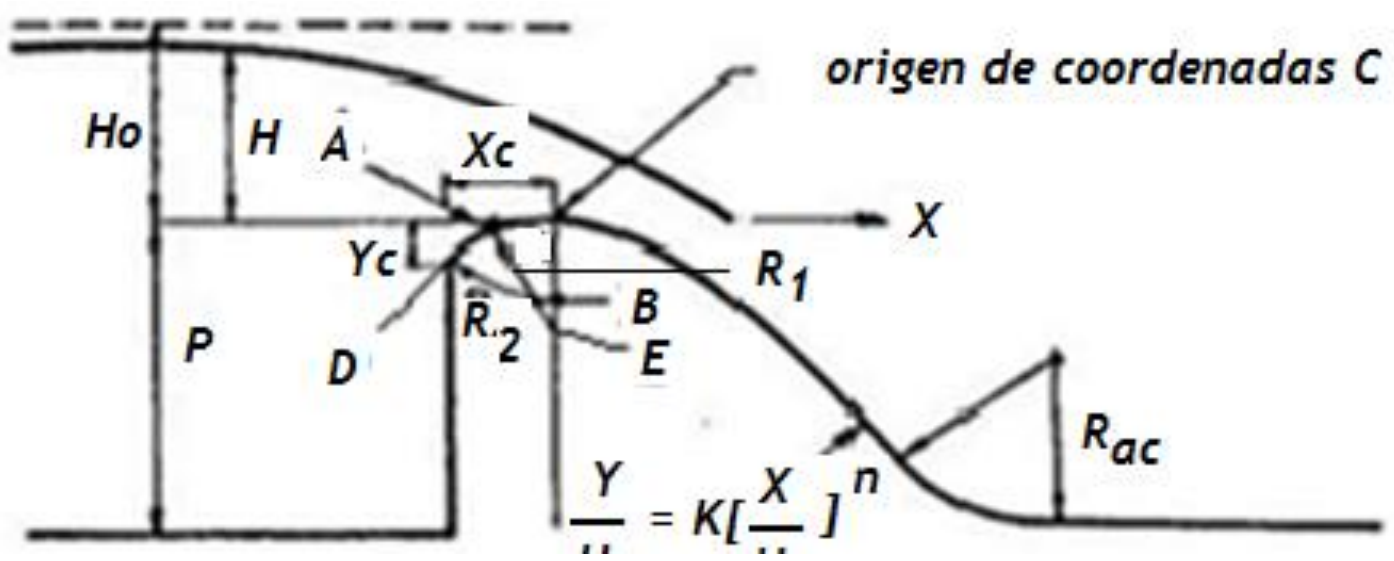

Figura 12. Determinación de la forma de la sección transversal del cimacio WES. Fuente: Diseño de presas pequeñas (USBR, 1987).

$L_{c}$ y $L_{p}$ tienen los significados arriba indicados.

Es evidente que si el número de inflexiones de entrada es igual al número de inflexiones de salida (Figura $11, a)(j=k$ ), la longitud a lo largo de la cresta del vertedor es igual a la longitud a lo largo del paramento superior $\left(L_{c}=L_{p}\right)$; mientras que si el laberinto no tiene los 
semiápices de salida de los extremos (Figura $11, \mathrm{~b}$ ), entonces $j-k=2$, y por tanto $L_{c}<L_{p}$.

Las longitudes $L_{c}$ y $L$ se relacionan teniendo en cuenta que la longitud de la cresta se afecta por las modificaciones que sufre la red de flujo en las proximidades de las inflexiones de entrada y salida, resultando:

$L=L_{c}-\left(j K_{w e}+k K_{w s}\right) H_{o}$

En la que:

$K_{\text {we }}$ : coeficiente que toma en cuenta la afectación en el vertimiento debido a la contracción de la red de flujo.

$K_{w s}$ : coeficiente que toma en cuenta la afectación en el vertimiento debido a la expansión de la red de flujo.

La obtención de los coeficientes $K_{w e}$ y $K_{w s}$ se logra mediante estudios experimentales en laboratorios especializados de hidráulica. El primero de ellos es fácil de obtener, pero en el caso del segundo se dificultan las condiciones de entrada del flujo, y por ello se define la existencia de un nuevo parámetro denominado $K_{w}=K_{w e}+K_{w s}$, el cual obviamente toma en consideración las afectaciones a la red de flujo debidas a la presencia de una inflexión de entrada y salida, es decir, la existencia de un semiciclo de un vertedor en laberinto, con lo que la Ecuación (6) se convierte en:

$L=L_{c}-n K_{w} H_{o}$ 
Tecnología y

Ciencias $₫$ Agua
2020, Instituto Mexicano de Tecnología del Agua

Open Access bajo la licencia CC BY-NC-SA 4.0

(https://creativecommons.org/licenses/by-nc-sa/4.0/)

En la que $n=$ número de semiciclos del laberinto, o lo que es lo mismo que:

$L=L_{c}-2 N K_{w} H_{o}$

Siendo $N$ el número de ciclos del vertedor de laberinto.

Para la obtención de los coeficientes $K_{w}$ y $K_{w e}$ se hicieron estudios experimentales en modelos físicos con las configuraciones que se presentan en la Figura 13.

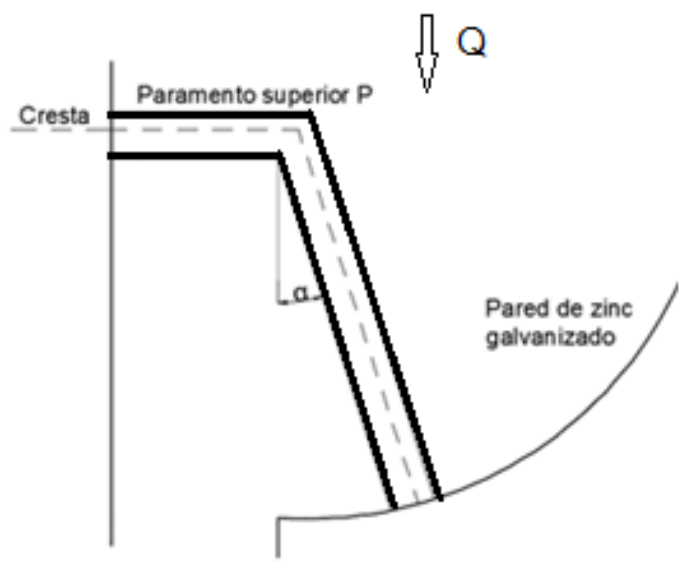

(a)

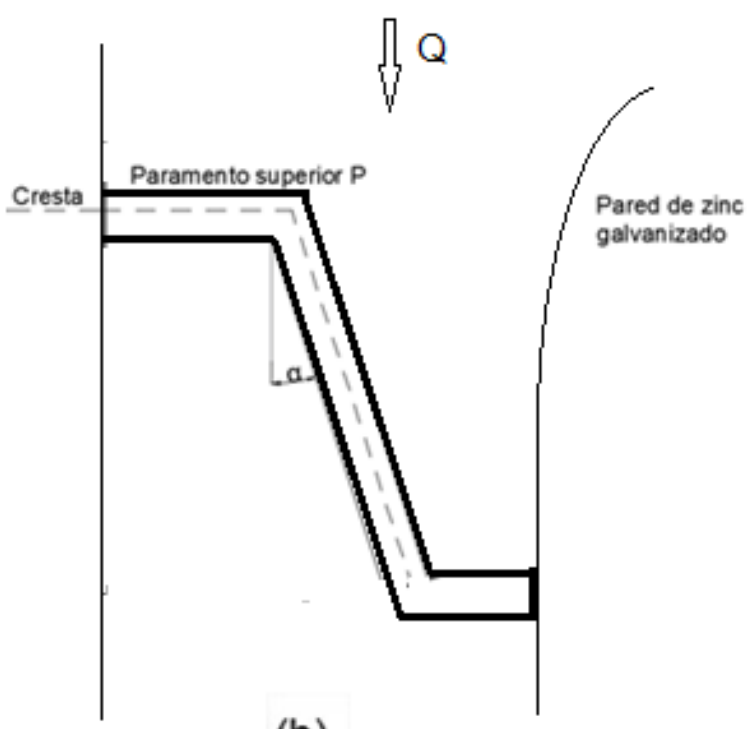

(b)

Figura 13. a) Entrada convergente de la red de flujo para obtener $K_{w e}$; b) semiciclo que incluye contracción de entrada y expansión de salida de la red de flujo para obtener $K_{w}$. 
En dichos estudios experimentales se tuvo en cuenta la variación del ángulo de cierre de los ciclos de entrada y salida, $a$, la variación de la altura relativa del paramento superior $P / H_{0}$ del vertedor y la variación de la carga hidráulica total relativa $H_{e} / H_{0}$ sobre el vertedor.

\section{Etapa 3. Mediciones y resultados experimentales}

- Relativas a los estudios para determinar la longitud mínima del ápice de entrada. Mediante estudios experimentales se pudo identificar la longitud mínima real $A_{\text {mín, }}$ expresada mediante la Ecuación (8). En estos estudios se tuvo que:

$Q=0.0528 \mathrm{~m}^{3} / \mathrm{s} ; P=0.20 \mathrm{~m} ; H_{0}=0.072 \mathrm{~m}$

Para los cuales se determinó que $X_{\text {vuelo }}=0.18 \mathrm{~m}$ y $h_{c}=0.017 \mathrm{~m}$ (Figura 8), resultando ello que el ancho mínimo teórico del ápice resulta $A_{\text {mín-teórico }}=2 *(0.18+0.017)=0.394 \mathrm{~m}$; pero en los ensayos realizados se pudo observar que $A_{\text {mín-real }}=0.34 \mathrm{~m}$ no refleja afectaciones por concepto de interferencia de láminas, lo cual se puede explicar por el hecho de que el contacto de ambas láminas de los vertimientos laterales tiene lugar a una distancia vertical desde la cresta 
tal que no afecta la capacidad de evacuación y definitivamente la $A_{\text {mín-real }}$ es la obtenida según la Ecuación (9), donde se consideraron los valores $A_{\text {min-real }}=0.34 \mathrm{~m}$ y $H_{0}=0.072 \mathrm{~m}$ :

$$
\frac{A_{\min }}{H_{o}}=4.722
$$

El empleo de la Ecuación (9) también se recomienda para laberinto con ángulo central $a>0^{\circ}$, donde se estará del lado de la seguridad, pues para esos otros valores del ángulo, la interferencia de láminas disminuye con respecto a aquellos de configuración rectangular $\left(a=0^{\circ}\right)$.

Finalmente, es oportuno e importante indicar que en todos los estudios experimentales realizados se pudo confirmar que cada una de las herramientas aplicables en cimacios WES frontales se pueden emplear cuando la configuración es en laberinto, gracias a la introducción del concepto de longitud efectiva arriba indicado; es decir, el coeficiente de gasto $m$ se obtiene a partir de las ecuaciones (10) a (14) y el apoyo de la Tabla 1 (Pardo \& Alegret, 2012), que se obtuvieron mediante ajuste matemático con el software profesional Table Curve 2D:

$m_{o}=\frac{a+c\left(P / H_{0}\right)^{0,5}+e\left(P / H_{0}\right)^{1}}{1+b\left(P / H_{0}\right)^{0,5}+d\left(P / H_{0}\right)^{1}+f\left(P / H_{0}\right)^{1,5}}$ 
Tecnología y

Ciencias $\stackrel{\varpi}{\varpi}$ Agua
2020, Instituto Mexicano de Tecnología del Agua

Open Access bajo la licencia CC BY-NC-SA 4.0

(https://creativecommons.org/licenses/by-nc-sa/4.0/)

$\sigma_{1}=\frac{a+c\left(H_{e} / H_{0}\right)^{2}+e\left(H_{e} / H_{0}\right)^{4}+g\left(H_{e} / H_{0}\right)^{6}+i\left(H_{e} / H_{0}\right)^{8}+k\left(H_{e} / H_{0}\right)^{10}}{1+b\left(H_{e} / H_{0}\right)^{2}+d\left(H_{e} / H_{0}\right)^{4}+f\left(H_{e} / H_{0}\right)^{6}+h\left(H_{e} / H_{0}\right)^{8}+j\left(H_{e} / H_{0}\right)^{10}}$

$\sigma_{2}=a+b\left(P / H_{e}\right)+c\left(P / H_{e}\right)^{2}+d\left(P / H_{e}\right)^{3}+e\left(P / H_{e}\right)^{4}+f\left(P / H_{e}\right)^{5}+g\left(P / H_{e}\right)^{6}+$

$h\left(P / H_{e}\right)^{7}+i\left(P / H_{e}\right)^{8}$

$\sigma_{3}=\frac{a+c\left(P^{*} / H_{O}\right)^{0,5}+e\left(P^{*} / H_{O}\right)^{1}+g\left(P^{*} / H_{O}\right)^{1,5}+i\left(P^{*} / H_{O}\right)^{2}+k\left(P^{*} / H_{O}\right)^{2,5}}{1+b\left(P^{*} / H_{O}\right)^{0,5}+d\left(P^{*} / H_{O}\right)^{1}+f\left(P^{*} / H_{O}\right)^{1,5}+h\left(P^{*} / H_{O}\right)^{2}+j\left(P^{*} / H_{O}\right)^{2,5}}$

$\sigma_{4}=a+b\left(h_{d} H_{e}\right)^{0,5}+c\left(h_{d} / H_{e}\right)^{1}+d\left(h_{d} H_{e}\right)^{1,5}+e\left(h_{d} H_{e}\right)^{2}+\left(h_{d} / H_{e}\right)^{2,5}+$

$g\left(h_{d} /_{H_{e}}\right)^{3}+h\left(h_{d} H_{e}\right)^{3,5}+i\left(h_{d} H_{e}\right)^{4}+j\left(h_{d} H_{e}\right)^{4,5}+k\left(h_{d} H_{e}\right)^{5}$

Tabla 1. Parámetros de las ecuaciones para obtener el coeficiente de gasto de cimacios WES.

\begin{tabular}{|c|c|c|c|c|c|c|c|}
\hline \multirow[t]{2}{*}{ Par. } & \multirow[t]{2}{*}{$\boldsymbol{m}_{\mathrm{o}}$} & \multirow[t]{2}{*}{$\sigma_{1}$} & \multicolumn{3}{|c|}{$\sigma_{2}$} & \multirow[t]{2}{*}{$\sigma_{3}$} & \multirow[t]{2}{*}{$\sigma_{4}$} \\
\hline & & & $1: 3$ & $2: 3$ & $3: 3$ & & \\
\hline$a$ & 0.38500865 & 0.79532682 & 1.0718077 & 1.1046593 & 1.0170604 & 0.76999623 & $\begin{array}{c}- \\
1.7440117 \mathrm{E}- \\
07\end{array}$ \\
\hline b & -0.57094102 & 42.053364 & $\begin{array}{c}- \\
0.86360153\end{array}$ & $\begin{array}{c}- \\
0.98692694\end{array}$ & 0.34980623 & -3.4930933 & -117.7002 \\
\hline c & $\begin{array}{c}- \\
0.090121694\end{array}$ & 36.833604 & 4.8324647 & 5.0263645 & $\begin{array}{c}- \\
2.27911451\end{array}$ & -2.5739119 & 2330.9643 \\
\hline d & 0.98200007 & -53.5648 & -14.65083 & -14.113728 & 6.3025867 & 5.7537742 & -19466.272 \\
\hline
\end{tabular}


Tecnología y

Ciencias $₫$ Agua
2020, Instituto Mexicano de Tecnología del Agua

Open Access bajo la licencia CC BY-NC-SA 4.0

(https://creativecommons.org/licenses/by-nc-sa/4.0/)

\begin{tabular}{|c|c|c|c|c|c|c|c|}
\hline $\mathbf{e}$ & 0.3699558 & -32.602056 & 26.092897 & 23.191671 & -10.009472 & 4,3302579 & 91701.717 \\
\hline $\mathbf{f}$ & - & -90.395781 & -28.096603 & -22.886658 & 9.7097979 & -4.0658495 & -268994.47 \\
\hline $\mathbf{g}$ & & -117.1965 & 17.975007 & 13.334193 & -5.6749561 & -3.0008521 & 510550.08 \\
\hline $\mathbf{h}$ & & 135.79851 & -6.282566 & -4.2218639 & 1.8342862 & - & -628467.79 \\
& & & & & & 0,82004921 & \\
\hline $\mathbf{i}$ & & 147.01894 & 0.92306655 & 0.55943426 & - & -1.2041974 & 484947.2 \\
\hline $\mathbf{j}$ & & & & & 0.25174542 & & \\
\hline $\mathbf{k}$ & & -16.394959 & & & & 1.951537 & -213308.96 \\
\hline
\end{tabular}

- Relativas a los estudios para determinar las longitudes características del vertedor. Al determinar los coeficientes $K_{w}$ y $K_{w e}$ que toman en cuenta las afectaciones en el vertimiento debido a las modificaciones de la red de flujo, se consideró lo siguiente: la variación del ángulo de cierre de los ciclos de entrada y salida; $a$, la variación de la altura relativa del paramento superior $P / H_{0}$ del vertedor, y la variación de la carga hidráulica total relativa $H_{e} / H_{0}$ sobre éste.

Los valores e intervalos estudiados para cada una de las tres variables mencionadas fueron los siguientes: $a: 0^{\circ}, 6^{\circ}, 8^{\circ}, 10^{\circ}, 12^{\circ}, 15^{\circ}$, $20^{\circ}$ y $35^{\circ}$, que son los mismos que se estudiaron en la mayoría de las publicaciones especializadas sobre vertedores de laberinto:

$P / H_{0}: 1$ a 3

$H_{e} / H_{0}: 0.4$ a 1.2 
Tecnología y

Ciencias $₫$ Agua
2020, Instituto Mexicano de Tecnología del Agua

Open Access bajo la licencia CC BY-NC-SA 4.0

(https://creativecommons.org/licenses/by-nc-sa/4.0/)

Los resultados obtenidos experimentalmente se presentan en las Tabla 2 y Tabla 3, donde se muestra la tendencia de la variación de los coeficientes $K_{w e}$ y $K_{w}$ según el ángulo a y el parámetro $\left(P / H_{0}\right)$.

Tabla 2. Ajuste de los valores medidos: $K w e=f(a, P / H o)$. Fuente: Borroto (2018).

\begin{tabular}{|c|c|c|c|c|c|c|c|c|c|}
\hline \multirow{2}{*}{$\boldsymbol{P} / \boldsymbol{H}_{\mathbf{o}}$} & \multicolumn{9}{|c|}{$\mathbf{a}$} \\
\cline { 2 - 9 } & $\mathbf{0}$ & $\mathbf{6}$ & $\mathbf{8}$ & $\mathbf{1 0}$ & $\mathbf{1 2}$ & $\mathbf{1 5}$ & $\mathbf{2 0}$ & $\mathbf{3 5}$ & $\mathbf{9 0}$ \\
\hline $\mathbf{1}$ & 1.82 & 1.67 & 1.58 & 1.52 & 1.48 & 1.44 & 1.27 & 1.07 & 0 \\
\hline $\mathbf{1 . 2}$ & 1.47 & 1.33 & 1.23 & 1.17 & 1.13 & 1.10 & 0.92 & 0.72 & 0 \\
\hline $\mathbf{1 . 4}$ & 1.39 & 1.24 & 1.15 & 1.09 & 1.05 & 1.02 & 0.84 & 0.64 & 0 \\
\hline $\mathbf{1 . 6}$ & 1.35 & 1.21 & 1.12 & 1.05 & 1.02 & 0.98 & 0.80 & 0.60 & 0 \\
\hline $\mathbf{1 . 8}$ & 1.30 & 1.15 & 1.06 & 1.00 & 0.96 & 0.92 & 0.75 & 0.55 & 0 \\
\hline $\mathbf{2}$ & 1.24 & 1.09 & 1.00 & 0.94 & 0.90 & 0.86 & 0.69 & 0.48 & 0 \\
\hline $\mathbf{2 . 2}$ & 1.22 & 1.07 & 0.98 & 0.91 & 0.88 & 0.84 & 0.66 & 0.46 & 0 \\
\hline $\mathbf{2 . 4}$ & 1.25 & 1.10 & 1.01 & 0.94 & 0.91 & 0.87 & 0.69 & 0.49 & 0 \\
\hline $\mathbf{2 . 6}$ & 1.25 & 1.10 & 1.01 & 0.95 & 0.91 & 0.88 & 0.70 & 0.50 & 0 \\
\hline $\mathbf{2 . 8}$ & 1.00 & 0.86 & 0.77 & 0.70 & 0.66 & 0.63 & 0.45 & 0.25 & 0 \\
\hline
\end{tabular}

Tabla 3. Ajuste de los valores medidos: $K w=f\left(a, P / H_{0}\right)$. Fuente: Borroto (2018). 
2020, Instituto Mexicano de Tecnología del Agua

Tecnología y

Ciencias $\stackrel{\varpi}{\triangleleft}$ Agua
Open Access bajo la licencia CC BY-NC-SA 4.0

(https://creativecommons.org/licenses/by-nc-sa/4.0/)

\begin{tabular}{|c|c|c|c|c|c|c|c|c|c|}
\hline \multirow{2}{*}{$\boldsymbol{P} / \boldsymbol{H}_{\mathbf{o}}$} & \multicolumn{9}{|c|}{$\mathbf{0}$} \\
\cline { 2 - 10 } & $\mathbf{0}$ & $\mathbf{6}$ & $\mathbf{8}$ & $\mathbf{1 0}$ & $\mathbf{1 2}$ & $\mathbf{1 5}$ & $\mathbf{2 0}$ & $\mathbf{3 5}$ & $\mathbf{9 0}$ \\
\hline $\mathbf{1}$ & 2.770 & 2.282 & 2.258 & 2.194 & 2.088 & 1.906 & 1.888 & 1.177 & 0 \\
\hline $\mathbf{1 . 2}$ & 2.800 & 2.312 & 2.288 & 2.225 & 2.118 & 1.936 & 1.918 & 1.207 & 0 \\
\hline $\mathbf{1 . 4}$ & 2.672 & 2.184 & 2.160 & 2.096 & 1.990 & 1.808 & 1.789 & 1.078 & 0 \\
\hline $\mathbf{1 . 6}$ & 2.535 & 2.047 & 2.023 & 1.959 & 1.853 & 1.671 & 1.652 & 0.941 & 0 \\
\hline $\mathbf{1 . 8}$ & 2.444 & 1.956 & 1.932 & 1.868 & 1.762 & 1.580 & 1.561 & 0.850 & 0 \\
\hline $\mathbf{2}$ & 2.390 & 1.902 & 1.878 & 1.814 & 1.708 & 1.526 & 1.508 & 0.797 & 0 \\
\hline $\mathbf{2 . 2}$ & 2.334 & 1.846 & 1.822 & 1.758 & 1.652 & 1.470 & 1.452 & 0.741 & 0 \\
\hline $\mathbf{2 . 4}$ & 2.235 & 1.747 & 1.723 & 1.659 & 1.553 & 1.371 & 1.352 & 0.641 & 0 \\
\hline $\mathbf{2 . 6}$ & 2.083 & 1.595 & 1.571 & 1.508 & 1.401 & 1.219 & 1.201 & 0.490 & 0 \\
\hline $\mathbf{2 . 8}$ & 1.934 & 1.446 & 1.422 & 1.358 & 1.252 & 1.070 & 1.052 & 0.341 & 0 \\
\hline
\end{tabular}

Una alternativa a la Tabla 2 es la Ecuación (15), que se presenta a continuación. Esta ecuación, con los correspondientes coeficientes de ajuste que se incluyen, se obtuvo mediante el software profesional Table Curve $3 D$, en donde se definió como variable dependiente a $K_{w e}$ y como variables independientes a $a$ y $P / H_{o}$ :

$K_{w e}=a+b \alpha+c \alpha^{2}+d \alpha^{3}+e \alpha^{4}+f \alpha^{5}+g\left(\frac{P}{H_{o}}\right)+h\left(\frac{P}{H_{o}}\right)^{2}+i\left(\frac{P}{H_{o}}\right)^{3}+j\left(\frac{P}{H_{o}}\right)^{4}+$ $k\left(\frac{P}{H_{o}}\right)^{5}$ 
Tecnología y

Ciencias $₫$ Agua
2020, Instituto Mexicano de Tecnología del Agua

Open Access bajo la licencia CC BY-NC-SA 4.0

(https://creativecommons.org/licenses/by-nc-sa/4.0/)

Siendo:

$a=32.505286$.

$b=0.05268624$.

$c=-0.024381393$.

$d=0.002470112$.

$e=-9.9482302 \mathrm{E}-05$.

$f=1.3451675 \mathrm{E}-06$.

$g=-87.736584$.

$h=97.801885$.

$i=-53.683096$.

$j=14.458445$.

$k=-1.527292$.

Coeficiente de determinación $r^{2}=0.9525$.

Alternativa a la Tabla 3 es la Ecuación (16), que se presenta a continuación; se obtuvo de forma semejante a la Ecuación (15):

$K_{w}=a+b \alpha+c \alpha^{2}+d \alpha^{3}+e \alpha^{4}+f \alpha^{5}+g\left(\frac{P}{H_{o}}\right)+h\left(\frac{P}{H_{o}}\right)^{2}+i\left(\frac{P}{H_{o}}\right)^{3}+j\left(\frac{P}{H_{o}}\right)^{4}+$

$k\left(\frac{P}{H_{o}}\right)^{5}$

$a=-15.645276$

$b=-0.26779196$. 
$c=0.056137767$.

$d=-0.005346804$

$e=0.000212062$.

$f=-2.85539 \mathrm{E}-06$.

$g=53.848291$

$h=-59.99205$.

$i=31.978292$.

$j=-8.2428759$.

$k=0.8239665$.

Coeficiente de determinación $r^{2}=0.9715$.

En la Ecuación (15) y Ecuación (16), el ángulo a se expresa en grados.

Las limitaciones para el empleo de la Tabla 2 y Tabla 3, así como sus correspondientes ecuaciones son las siguientes:

1. Solo válidas para el rango $0 \leq a \leq 35$.

2. Para diseños con $P / H_{0}>2.8$ se deben emplear los valores de $K_{w}$ y $K_{\text {we }}$ correspondientes a $P / H_{o}=2.8$.

3. Para el empleo específico de las tablas es aconsejable seleccionar para el ángulo a uno de los valores exactos presentados al inicio de las mismas; mientras que empleando las ecuaciones correspondientes será válida la interpolación. 


\section{Ejemplo para comparar resultados de cálculos siguiendo los criterios de diseño de Crookston (2010) con los del autor del presente artículo}

Datos:

$Q=800 \mathrm{~m}^{3} / \mathrm{s}$.

$H_{\mathrm{o}}=H_{T}=3 \mathrm{~m}$.

Altura de paramento superior $P=9.9 \mathrm{~m}$.

Ángulo central $a=35^{\circ}$.

Número de ciclos $N=1$.

Obtener la longitud total vertedora a construir.

Según Crookston, con $a=35^{\circ}$ y $H_{T} / P=0.3$, y cresta un cuarto redondeada, se obtiene $C_{d}=0.7$ (correspondería $m=0.46$ ) resultando $L_{c}=75.22 \mathrm{~m}$.

Según este autor, con $a=35^{\circ}$ y $P / H_{0}=3.3$, y cresta tipo WES truncada, se obtiene $m=0.49$, resultando $L=70.93 \mathrm{~m}$ y $L_{c}=72.98 \mathrm{~m}$.

Se aprecia la necesidad de una menor longitud vertedora total para el laberinto con WES truncado debido a su mayor eficiencia hidráulica, léase coeficiente de gasto. 
Tecnología y

Ciencias $\stackrel{\Im}{\Im}$ Agua
2020, Instituto Mexicano de Tecnología del Agua

Open Access bajo la licencia CC BY-NC-SA 4.0

(https://creativecommons.org/licenses/by-nc-sa/4.0/)

\section{Conclusiones}

1. Los resultados experimentales confirman la validez del empleo de los vertedores tipo WES truncados en los aliviaderos con configuración tipo laberinto, con total seguridad de la no afectación del coeficiente de gasto.

2. La introducción de los coeficientes de contracción de la red de flujo $K_{w e}$ y $K_{w}$ permiten el empleo de la longitud efectiva en la ecuación fundamental del aliviadero con configuración en laberinto. Para ello deberán respetarse los rangos experimentados de las variables hidráulicas principales; esto es, ángulo $a$, altura relativa del paramento superior $P / H_{0}$ y cargas relativas $H_{e} / H_{0}$, así como las limitaciones definidas para las tablas y ecuaciones con las cuales se obtienen los coeficientes mencionados.

3. El uso de la longitud mínima para los ápices de entrada y de salida (excluyendo los extremos) garantiza la eliminación de la interferencia de láminas y evita la sumergencia de los vertedores con configuración en laberinto.

4. El funcionamiento de los ciclos de salida sin interferencia de láminas ni sumergencia hace que en ellos el régimen de circulación por lo general sea supercrítico, lo cual requiere de nuevos estudios experimentales para su adecuado diseño. Esta etapa de investigaciones ya está en marcha en el Centro de Investigaciones Hidráulicas de la Universidad Tecnológica de La Habana "José Antonio Echeverría", Cuba. 
Teçnología y

Ciencias $₫$ Agua
2020, Instituto Mexicano de Tecnología del Agua

Open Access bajo la licencia CC BY-NC-SA 4.0

(https://creativecommons.org/licenses/by-nc-sa/4.0/)

\section{Agradecimientos}

El autor del presente trabajo desea expresar sus agradecimientos a Reinaldo Pagés Lamas y Anabel Borroto Roche, estudiantes de quinto año de la Carrera de Ingeniería Hidráulica en la Universidad Tecnológica de La Habana, pues con su dedicación al culminar sus respectivas tesis de grado en los años 2017 y 2018 lograron realizar los trabajos experimentales que posibilitaron los resultados que aquí se presentan. Se reconoce también el trabajo de la ingeniera Susel Lobaina Fernández, cotutora de los mismos.

\section{Referencias}

Borroto-Roche, A. (2018). Modificaciones de la red de flujo para una entrada convergente y una salida divergente en aliviaderos de laberinto con cimacio tipo WES truncado (tesis de grado). Universidad Tecnológica de La Habana, "José Antonio Echeverría" Cujae, La Habana, Cuba.

Carralero, I. (2016). Influencia del truncado en la capacidad de evacuación de cimacios tipo PPSV y PPCV (tesis de grado). Universidad Tecnológica de La Habana, "José Antonio Echeverría" Cujae, La Habana, Cuba.

Crookston, B. M. (2010). Labyrinth Weirs (tesis de grado). Recuperado de http://digitalcommons.usu.edu/etd/802 
Tecnología y

Ciencias $₫$ Agua
2020, Instituto Mexicano de Tecnología del Agua

Open Access bajo la licencia CC BY-NC-SA 4.0

(https://creativecommons.org/licenses/by-nc-sa/4.0/)

Pagés-Lamas, R. (2017). Modelo físico 3D de vertedor de laberinto (tesis de grado). Universidad Tecnológica de La Habana, "José Antonio Echeverría" Cujae, La Habana, Cuba.

Pardo, R., \& Alegret, E. (2012). Diseño hidráulico de aliviaderos y obras de toma para presas. La Habana, Cuba: Editorial Félix Varela.

Pardo-Gómez, R. (2019). El empleo de cimacios tipo WES o elípticos en vertedores tipo laberinto. Parte I. Tecnología y ciencias del agua, 10(3), 1-11. DOI: 10.24850/j-tyca-2019-03-01

Tullis, B. P., Asce, M., Young, J. C., \& Chandler, M. A. (2007). Headdischarge relationships for submerged labyrinth weirs. Journal of Hydraulic Engineering, 133(3).

USBR, United States Bureau of Reclamation. (1987). Design of small dams ( $3^{\text {rd }}$ ed.). Denver, USA: Water Resources Technical Publication. 